\title{
Detecting and treating hypothyroidism during pregnancy
}

\author{
P Reed Larsen
}

Much has been written about the diagnosis and treatment of hypothyroidism in pregnant women. We know that the fetus requires $T_{4}$ for normal development, and that the function of the fetal thyroid gland is not fully established until midgestation. Until then, the mother is the only source of $T_{4}$. We also know that there is a $30-40 \%$ increase in maternal $T_{4}$ secretion beginning as early as the sixth week of gestation and that there may be more complications of pregnancy in women with hypothyroidism.

How often hypothyroidism begins during pregnancy is not known, but women with previously diagnosed hypothyroidism (about $1 \%$ of pregnant women) who have not had their levothyroxine dose increased might redevelop this. A recent large study by Casey et al. (Obstet Gynecol 105: 239-245) found that previously unrecognized overt hypothyroidism (high serum TSH and low serum free $\mathrm{T}_{4}$ values) and subclinical hypothyroidism (high serum TSH but normal serum free $\mathrm{T}_{4}$ ) occur in approximately $0.2 \%$ and $2.3 \%$ of pregnancies, respectively; however, pregnancy-induced changes in serum proteins might make automated measurements of serum free $T_{4}$ unreliable. I mention this because Casey et al. found that $20 \%$ of the subclinical hypothyroid women had serum TSH values $>10 \mathrm{mU} / \mathrm{l}$, and that in the subclinical group there was a twofold higher incidence of preterm delivery, a threefold increase in placental abruption, and a significant increase in the proportion of neonates admitted to the intensive-care unit. These findings suggest a more significant deficiency. All told, each year $1-2 \%$ of the $\sim 4$ million pregnant women in North America are at risk of hypothyroidism.

Clinicians agree that, during pregnancy, women with newly diagnosed overt hypothyroidism or those already being treated for it should have their serum TSH concentrations measured monthly and normalized by administration of levothyroxine in appropriate
I believe we should make every effort to discover and treat primary hypothyroidism during pregnancy

$P R$ Larsen is the Editor-in-Chief of Nature Clinical Practice Endocrinology \& Metabolism, Chief of the Division of Endocrinology, Diabetes and Hypertension at Brigham and Women's Hospital and a Professor of Medicine at Harvard Medical School, Boston, MA, USA.

\section{Competing interests}

The author declared he has no competing interests.

www.nature.com/clinicalpractice doi:10.1038/ncpendmet0114 doses ( $2 \mu \mathrm{g} / \mathrm{kg} /$ day ideal body weight). There is disagreement about the subclinical hypothyroidism group. Some argue that levothyroxine should not be given since no randomized outcome trials of its use in pregnant women with subclinical hypothyroidism have been performed. Several professional organizations advocate such trials but only one prospective study is underway, and it will probably be years before it reports.

Meantime, what should clinicians do? I believe we should make every effort to discover and treat primary hypothyroidism during pregnancy. This includes educating women already receiving levothyroxine (especially those with severe thyroid deficiency) about increasing their dose early in gestation. We should ensure that pregnant women in regions of iodine deficiency receive appropriate supplementation. We should pursue aggressive case-finding in high-risk women such as those with goiter, symptoms or signs suggesting hypothyroidism, a family history of autoimmune thyroid disease or type I diabetes. The earlier in gestation this is done the better, so this testing would be best done by the primary-care physician or endocrinologist rather than the obstetrician. Pregnant women with hypothyroidismsomewhat arbitrarily defined as a verified $\mathrm{TSH}>5 \mathrm{mU} / \mathrm{l}$, with a normal or low free $\mathrm{T}_{4}$ value - should be advised of the potential risks and benefits of levothyroxine therapy. I favor initiating levothyroxine therapy, monitoring monthly serum TSH values and maintaining levels in the normal range for pregnancy, remembering that a significant excess of levothyroxine could have adverse effects on the pregnancy and should be avoided. Although this recommendation for levothyroxine treatment is not evidence based, I am concerned about the outcomes in the large numbers of pregnancies that will occur before we have satisfactory data. 